\title{
Economía informal: un análisis al comercio ambulante de la región del Maule, Chile
}

Miguel A. Bustamante

Universidad de Talca, Chile / mabu@utalca.cl

Ricardo Alejandro Díaz Pérez

Universidad de Talca, Chile / ricadip@yahoo.com

Patricia Villarreal Navarrete

Universidad de Talca, Chile / paty_villarreal74@hotmail.com

Recibido: 8 de octubre de 2009

Aceptado: 20 de mayo de 2009

\section{RESUMEN}

El presente trabajo caracteriza el comercio informal de la Región del Maule, Chile. Identifica causas, limitaciones, motivaciones de sus participantes así como también describe el cumplimiento de regulaciones legales, sanitarias y laborales. Se aplicó un cuestionario estructurado a una muestra aleatoria en seis ciudades que incluyó cuatro capitales provinciales y dos ciudades de tamaño medio. Se concluye que la mayoría posee un bajo nivel educacional con edades que promedian los 45 años, acreditan algún tipo de permiso municipal y no cuentan con las mínimas condiciones de sanidad y laborales. Sin embargo, aunque enfrentan una situación de desamparo social, no cambiarían su actual fuente laboral lo que confirma que el comercio informal es, para ellos, un estilo de vida.

Palabras clave: Economía informal, economía subterránea, comercio informal.

\section{ABSTRACT}

The present work characterizes the informal business of the Maule Region in Chile. It identifies causes, limitations and participants' motivations as well as compliance with legal, sanitary and labor regulations. A questionnaire was administered to a random sample in six cities that included four provincial capitals and two medium size cities. The survey reveals that the majority of the participants have a low educational background, an average age of 45 years, possess some type of municipal permit, and 
do not have the minimal work and health conditions. Nonetheless, although they face social neglect, the participants expressed no desire to change their present labor situation which confirms that the informal economy is their life style.

Keywords: Informal economy, underground economy, informal commerce.

\section{INTRODUCCIÓN}

En sus diversas expresiones, la economía informal tiene su origen en el escaso capital humano y en la abundancia de recursos naturales que estimulan especializaciones en sectores de baja productividad y estancan el crecimiento (Brunner y Elecqua, 2003) el que se fundamenta en el modelo neoclásico de Solow (1956) y en la teoría de crecimiento endógeno de Lucas (1988) (citados en Oroval, E. y Escardíbul, J. 2000), coincidiendo en que para aumentarlo son esenciales el capital físico y el progreso tecnológico y que, como ya había observado Adam Smith en el siglo XVIII, éste incluye "las facultades productivas adquiridas y útiles de todos los habitantes de un país" (Brunner y Elacqua, 2003).

Se reconoce de esta forma que el capital humano, descrito como el conjunto de las capacidades productivas que un individuo adquiere por acumulación de conocimientos generales, es vital en la economía. Sin embargo, sus falencias explican también el desarrollo de expresiones comerciales que contraen la riqueza (Cruz P., Rojas S., Vega G., 2002) lo que afecta, entre otros, a los segmentos poblacionales que se desempeñan en la economía informal.

Por otra parte, las teorías del capital humano (Mincer, 1958, Schultz, 1961 y Becker 1964; citados en Oroval y Escardíbul, op. cit) reconocen a la educación como una inversión que aumenta la dotación de capital (humano), incrementa la productividad, los ingresos futuros y establece una relación causal entre educación, productividad e ingresos, de manera que, niveles inferiores de educación implican menores nivel de renta y contracción del crecimiento económico. Complementariamente algunas teorías del crecimiento consideran vitales el capital físico y el trabajo (Solow, 1956) indicando que el progreso tec- 
nológico da origen a una "nueva teoría del crecimiento económico" denominada de "crecimiento endógeno" (Lucas, 1988 y otros autores; citados en Oroval, E. y Escardíbul, J. 2000) según la cual la economía informal pone en evidencia disyunciones de la economía.

En lo específico, economía informal, sector informal, sector residual, economía subterránea, no tradicional, no moderna, contra-económica, economia popular, alternativa, marginal, autónoma, clandestina, paralela, invisible, ilegal y oculta (http://www.ilo.org/Search3/searchOnFast.do, OIT (a, b, c, d, e), 2002), entre otras denominaciones, son las adoptadas para referirse a las diversas actividades económicas que surgen de inhabilidades de la economía y de las oportunidades de beneficio que logran algunos agentes sociales al actuar fuera de normas, reglamentos y leyes que los regulen (Lasso, 2000). Es un fenómeno analizado en la literatura económica que se hace presente tanto en países desarrollados como en vías de desarrollo (Rodríguez, 1988). Se incrementa en tiempos de crisis económica, sin embargo, es la expresión actualizada de tradiciones y costumbres ancestrales de la humanidad que ya existían en la época pre colombina en la forma de tianguis o comercios de medicinas, granos y tejidos, entre otros productos y servicios. Por supuesto, en aquel tiempo existía bajo la regulación de monarquías (Lasso, 2000) pero en la actualidad se realiza libremente, de manera espontánea y sin aportar ingresos tributarios al Estado (Tijerina y Medellín, 1990).

El debate en torno a la economía informal, desde el punto de vista "neutro", adquiere una perspectiva más bien estadística refiriéndose a prácticas "oscuras" siendo analizada en torno a términos tales como "trabajo decente y economía informal" (http://www.ilo.org/public/ spanish/region/ampro/cintefor/temas/informal/tdecpob/index. htm) adoptando el término de "Economía Informal" centrado en el trabajo informal y en las relaciones de trabajo y no en las características de las empresas (OIT (b,e), 2002). Sin embargo, se desarrolla desde mercados formales tales como la construcción, en los que el control y el registro se realizan en la informalidad amparada por gremios y políticos; un fenómeno que, por sus dimensiones, produce daño social y clientelismo (De Soto, 1992). 


\section{ECONOMÍA INFORMAL, BARRERAS Y BENEFICIOS}

En general, la economía informal propicia objetivos diferentes. Por una parte, beneficios derivados de ahorros por no pago de servicios (luz, agua, arriendo, entre otros) así como por la ausencia de contratos de trabajo y sus costos (vacaciones, seguridad social y seguridad laboral) caracterizándose por el no pago de impuestos (Diamond y Mirrless, 1971) y por la variabilidad de las relaciones laborales informales (OIT (a,c), 2003). Surge, en consecuencia, producto de ineficiencias de leyes así como de los altos costos de tiempo, dinero e información que implica la legalidad (Ghersi y De Soto, 1987).

Gestores de algunos servicios informales (INEGI, 1997), por una parte, acceden a ingresos disminuidos (Jusidman, 1993) sin seguros social, laboral, vacaciones, ni aguinaldos y, por otra, ahorran costos de permanencia, costos de acceso y, por supuesto, reparto de utilidades y el pago de tributos (Hernández, 1997). En general, las expresiones de esta informalidad dan cuenta de los trabajadores por cuenta propia, servicios y trabajos esporádicos, servicio doméstico, empleos informales en organizaciones formales, micro comercios y micro empresas informales, comercio ambulante y vendedores callejeros que se caracterizan por trabajar fuera de normas, regulaciones y leyes (Lasso, 2000).

Desde el punto de vista macroeconómico, la globalización, desregulación y reestructuración de procesos han permitido emplear mano de obra bajo contratos más flexibles (OIT (a,c), 2003) dando sentido a la expresión "no son informales los hombres sino más bien las actividades que estos realizan" (Gershi, 1987). Además, el comercio informal o ambulatorio es un fenómeno evidente en el mundo en la forma de comercios de calle al margen de regulaciones y disposiciones legales (Martínez, 1999).

Entre sus beneficios directos se mencionan la flexibilidad, autonomía e independencia relativa de sus participantes, demás, de algunos beneficios indirectos para los consumidores tales como: mayor acceso a la compra de productos, precios más bajos y, a veces, ciertas facilidades en el pago. Además, aunque desempeña un rol protagónico en ciertas clases sociales al facilitar el acceso a productos 
más baratos que en el mercado formal, influye negativamente en el desarrollo de la economía por la evasión de impuestos y la reducción de la recaudación fiscal (PREALC. 1988), lo que equivale a establecer una tasa impositiva sobre el sector formal induciendo bajo crecimiento y menor bienestar (Diamond y Mirrless, 1971). En consecuencia, una primera interpretación de causas y efectos indicaría que, si se flexibilizara la legislación laboral y simplificaran los requisitos para establecer un negocio, se incentivaría el sector formal de la economía (Tanzi, 1983). Este tipo de actividades requiere por una parte, los menores esfuerzos que exigen las actividades comerciales (inversión, infraestructura y capital) y, por otra, las menores exigencias desde el punto de vista legal (permisos, licencias sanitarias, entre otras).

\section{Economía informal en América Latina}

El concepto de "economía informal" se ha situado entre los años 1940 y 1981 (De Soto, 1992) a causa del crecimiento de la población activa, mayor participación de las mujeres en el empleo, incremento del éxodo hacia las ciudades y disminución del empleo en la economía formal; a nivel de los países americanos se expresa de diversas formas.

En general, los trabajadores por cuenta propia marcan una presencia que bordean el $25 \%$; en el comercio alcanzan a cerca de $30 \%$ y en general en los servicios llegan a un 47\%. En América Latina, el empleo "informal urbano" pasó, en el transcurso de la última década, del $52 \%$ al 58\% (OIT (a,c), 2003). Situación ratificada al proponer que "[e]l trabajo decente es la mejor política social y la mejor política económica. Sin embargo, esto no ha sido visto así a lo largo de la historia" (Pleitez, 2008).

En Chile, la fuerza de trabajo se ha incrementado a una tasa promedio de $3.9 \%$ creciendo en casi 549 mil nuevos empleos en los últimos tres años. Los crecimientos más rápidos del empleo se observan en las regiones Primera, Segunda, Quinta, Séptima, Décima, Décimo Segunda y Región Metropolitana (MIDEPLAN, CASEN. 2001 - 2006). El empleo informal equivale a un $22.3 \%$ de los ocupados y un $20.4 \%$ de la fuerza de trabajo. De los ocupados 
entre 15 y 24 años, un $16 \%$ se reconoce informal llegando a un $34 \%$ entre los 55 y 64 años y a un $41.9 \%$, en las edades superiores (MIDEPLAN; CASEN; 2001 - 2006), confirmando la precariedad del empleo a medida que aumenta la edad. Asimismo, bajo el título "Determinantes de la Movilidad de la Pobreza en Chile, 1996-2006" (Arzola y Castro, 2007), se pone en evidencia que el país, aunque avanza a nivel macro, se estanca relativamente sin haber sabido aprovechar las oportunidades que otros países han tornado en ventaja y les han permitido avanzar a pasos agigantados (Doing Business 2009, www.lyd.org).

A nivel internacional, se constata la importancia de la economía informal en términos de países y número de estudios.

Entre los países desarrollados (con una renta por habitante superior a 10.000 dólares anuales; una industria potente y tecnológicamente avanzada y un alto nivel de vida, entre otros aspectos), Estados Unidos presenta a esta fecha, 1339 estudios; Unión Europea 634; Canadá 609 y Japón 562.

Países de desarrollo intermedio tales como la Federación Rusa, cuenta a la fecha con 297 trabajos; Argentina 959; Chile 869; Brasil 916; México 865 y Malasia 343 estudios relacionados con el comercio informal, abordando temáticas bastante similares a las de los países desarrollados. Sin embargo, merecen un reconocimiento especial países subdesarrollados (caracterizados por una renta por habitante inferior a los 2.000 dólares anuales; un desarrollo industrial incipiente, dependiente de la inversión externa, basados en mano de obra batata y recursos naturales, deficiente infraestructura, elevados índices de analfabetismo y alto crecimiento demográfico, entre otros factores) como Haití que a la fecha presenta 292 estudios; Etiopía 254; Kenia 380 y Pakistán 363, trabajos en torno al comercio informal, mostrando, en términos relativos, un número inferior de estudios, pero que sin embargo abordan las mismas preocupaciones de los países más desarrollados (http://www.ilo.org/Search3/ searchOnFast.do).

En síntesis, a nivel global los investigadores abordan bajo la denominación Trabajo Decente, Trabajo y Formación, Trabajo y Pobreza, Estudios de Género, Análisis de Grupos Vulnerables, Trabajo Informal y Sindicalismo, entre otros títulos, el tema de la Economía Informal y sus de- 
rivados tales como el Comercio Informal y el Comercio Ambulante que motivan este trabajo.

\section{OBJetivos y mÉtodos}

El presente trabajo tiene el objetivo de caracterizar el comercio informal de la Región del Maule (Chile), identificar causas, limitaciones, motivaciones de sus participantes así como también describir el nivel socioeconómico promedio, el cumplimiento de regulaciones legales, sanitarias, laborales y la disposición relativa hacia la formalización de sus actividades.

El método de investigación contempló una fase exploratoria con el propósito de indagar, obtener y sistematizar información relevante. La fase descriptiva y concluyente de corte transversal permitió describir, medir y evaluar factores del comercio informal mediante la aplicación de un cuestionario (Kinnear y Taylor, 1993; Hernández, et. al. 1998).

El cuestionario fue sometido a pilotaje para eliminar defectos (PREALC, 1988) alcanzando un Alfa de Cronbach de 0,7252 para 22 ítems y de 0,6163 para 19 ítems, lo que le otorga un alto nivel de confiabilidad al estudio. Las preguntas se dividieron en dos partes, una orientada a obtener la información demográfica y la segunda para recoger antecedentes del negocio informal. Se utilizó preguntas "cerradas" para conocer actitudes generales y preguntas "dicotómicas" y de "selección múltiple" (Kinnear y Taylor, 1993) para la caracterización de los comercios. Su aplicación presencial, leída por el encuestador, contempló una duración de entre quince y treinta minutos. El porcentaje de rechazo fue inferior al $3 \%$.

La población objeto de estudio se determinó sobre la base de los registros que mantienen los respetivos municipios de los comercios informales, y contempló estratos mutuamente excluyentes, provenientes de las ciudades con mayor población, como las capitales provinciales de Cauquenes, Curico, Linares y Talca más las ciudades de Constitución y Parral (INE, Censo 2002). La muestra fue de tipo probabilística (Hernández, et. al, 1998) y se utilizó la variante estratificada aproporcional (Kinnear y Taylor, 1993).

El tamaño de la muestra consideró un nivel de confianza de $95 \%$ y un error estimado de $5 \%$ con varianza máxima. De esta 
forma la muestra total alcanzó a 258 elementos. El número total de cuestionarios aplicados fue de 80 en Talca, 39 en Curico, 37 en Linares, 34 en Cauquenes, 32 en Parral y 36 en Constitución. El análisis de datos se realizó con el programa computacional SPSS 11.5 y el SPSS 10.0.

El procedimiento de selección de los individuos fue un croquis de ubicación en cada ciudad; se consideró un procedimiento de reemplazo y como criterio de selección, se identificó al propietario o encargado de cada comercio.

\section{V.Resultados}

La Región del Maule se extiende entre los $34^{\circ} 41^{\prime}$ y los $36^{\circ}$ 33' de latitud Sur y desde los $70^{\circ} 20^{\prime}$ de longitud Oeste hasta el Océano Pacífico, con un clima mediterráneo. La región posee una superficie de $30.296,1 \mathrm{Km}^{2}$ y registra una población de 908.097 habitantes (Censo 2002). La fuerza de trabajo (según el último Censo, 2002) es de 303.000 personas, lo que equivale a un $33.366 \%$ de la población total, además un $66.4 \%$ corresponde a población urbana y la densidad alcanza 29.9 habitantes por $\mathrm{Km}^{2}$ (Tabla 1). Las principales actividades económicas son la agricultura (sector forestal, fruticultura de exportación y hortícola) y la industria (agroindustria, madera y los vinos) consideradas como las más dinámicas, generando la mitad del valor agregado industrial regional (http://www.conama. cl/ certificación/1142/propertyvalue-12142.html).

Tabla 1. Datos sociodemográficos de las ciudades en estudio, Censo 2002

\begin{tabular}{lccccccr} 
Ciudad & Cauquenes & Constitución & Curico & Linares & Parral & \multicolumn{1}{c}{ Talca } & Regional \\
\hline Superficie Km2 & $2,126.3$ & $1,343.6$ & $1,328.4$ & $1,465.7$ & $1,638.4$ & 231.5 & $30,296.1$ \\
Población censo 1992 & 10,279 & 40,340 & 104,113 & 77,316 & 38,067 & 171,287 & 836,141 \\
Población censo 2002 & 41,217 & 46,081 & 119,585 & 83,249 & 37,822 & 201,797 & 908,097 \\
Variación Ínter censal & 2.3 & 14.2 & 14.9 & 7.7 & -0.6 & 17.8 & 8.6 \\
Urbana & 30,771 & 37,202 & 100,506 & 68,224 & 26,397 & 193,755 & 603,020 \\
Rural & 10,446 & 8,879 & 19,079 & 15,025 & 11,425 & 8,042 & 30,5077 \\
Hombres & 20,092 & 23,389 & 58,768 & 40,518 & 18,963 & 96,810 & 452,988 \\
Mujeres & 21,125 & 22,692 & 60,817 & 42,731 & 18,859 & 104,987 & 455,109 \\
\hline
\end{tabular}

Fuente: Instituto Nacional de Estadística. Censo 2002, Mapa interactivo www.censo2002.cl. 
La población por género sigue el patrón nacional, sin embargo, se aprecia un repunte de la población masculina en Constitución en comparación con lo que sucede en Cauquenes y Parral y una cierta superioridad del número de mujeres en las ciudades de Talca y Linares. En general, predominan los hombres, entre 15-19 años y de nivel educativo bajo (básica incompleta y básica completa). El $29.4 \%$ de los ocupados acredita formación de nivel medio, un $42.9 \%$ nivel básico, un $8.8 \%$ señala poseer estudios universitarios y un $8.2 \%$ estudios medios técnico profesional.

Entre los años 1992 y 2002 la Región del Maule mantenía un importante porcentaje de desocupados, mayoritariamente hombres. Sin embargo, al año 2002 la Región es representada por las mujeres inactivas en relación con el total nacional (INE, CENSO 2002).

Entre el año 1998 y el actual la población económicamente activa (PEA) crece alrededor de 16.000 personas por año (Programa Chile Califica, 2007); se destruyen 3,630 puestos de trabajo en el periodo 1998-2003 incrementando el desempleo masculino. Al año 2007, los niveles de empleo de los hombres caen de 231 mil a 221 mil personas promedio anual. Sin embargo, el nivel de empleo femenino aumentó de 90 mil a 96 mil personas promedio por año. Para los grupos jóvenes las tasas de desempleo fueron particularmente altas alcanzando el 28\% (Programa Chile Califica, 2007).

La población de la Región del Maule varía de $6.0 \%$ a $6.2 \%$ del total nacional para los años 1992 y 2002 respectivamente (últimos dos Censos nacionales), mostrando un incremento sostenido a través del tiempo. La ciudad más poblada es Talca, con más del 20\% de la población de la Región, seguida de Curico con un 13\% y Linares con un $9 \%$. Cauquenes muestra un incremento explosivo a una tasa de más de $300 \%$.

En la actualidad las estadísticas nacionales muestran (trimestre julio-septiembre de 2008) una tasa de empleo de $92.2 \%$ y los desocupados $(7.8 \%)$ una distribución por género de $6.7 \%$ para los hombres y un $9.5 \%$ para las mujeres. En la Región del Maule, los ocupados alcanzan a $90.9 \%$ y de estos, los "por cuenta propia" llegan a $21.13 \%$ (91.570) mostrando una estimulante variación negativa de $2.5 \%$ respecto del trimestre anterior. La tasa de desocupados $(9.1 \%)$ es inferior al $12.3 \%$ del año 2007 , y se desagrega en cesantes $(7.9 \%)$ 
y quienes buscan trabajo por primera vez (1.2\%). La tasa de desempleo es de $8.8 \%$ para los hombres y de un $9.7 \%$ para las mujeres. Cauquenes y Curico alcanzan un $7.7 \%$, Talca llega a un $8.6 \%$ y Linares supera los dos dígitos con un $11.7 \%$ de desempleados (INE, 2008).

\section{TABLA 2: GÉnERO Y TIPO de ACTIVIDAD}

\begin{tabular}{cccccc} 
Género & Frecuencia & $\%$ & Tipo de Trabajo & Frecuencia & $\%$ \\
\hline Masculino & 130 & 50.4 & Comerciante & 251 & 97.3 \\
Femenino & 128 & 49.6 & Lustrabotas & 6 & 2.3 \\
Total & 258 & 100.0 & Flete & 1 & 0.4 \\
& & & Total & 258 & 100,0 \\
\hline
\end{tabular}

Fuente: Cuestionario final: antecedentes demográficos.

\section{CaRACTERIZACIÓN DE LOS PARTICIPANTES DEL COMERCIO INFORMAL}

El análisis demográfico (Tabla 2) permite afirmar que un 50,5\% corresponde al género masculino y el 49,5\% al femenino. El $97 \%$ son comerciantes y el $3 \%$ prestadores de servicios, grupo que incluye lustrabotas y fleteros.

En las ciudades de Talca, Curico y Parral, se aprecia que existe un mayor número de personas de sexo masculino a cargo del comercio informal o como responsable de la actividad, al contrario de quienes asumen esa función en la ciudad de Constitución, donde se evidenció predominio femenino. Sin perjuicio de lo señalado, puesto que $\left(X^{2}{ }_{c}<\right.$ $X^{2}{ }_{t}$, se acepta la hipótesis nula, lo cual indica que no existe una relación que discrimine entre las ciudades en estudio y el género de los entrevistados a cargo de los comercios $\left(X^{2}{ }_{C}=9,141, X_{0.95,5}\right.$ g.l. $\left.=11,071\right)$.

Gráfico nº 1: DISTRIBUCIÓN POR EDADES DE LA MUESTRA

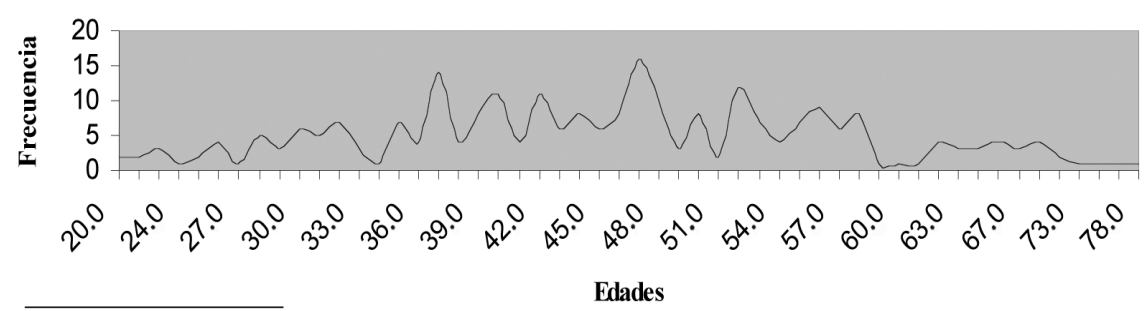

Fuente: Cuestionario final: antecedentes demográficos. 
La media de las edades (Gráfico 1) fue de 45 años y su moda es de 47. El porcentaje más bajo se encuentra entre los 20 y 25 años, y entre los 73 y 68 años alcanzando un $4 \%$ y $1.6 \%$ de presencia respectivamente. El estado civil de los encuestados (Tabla 3) refleja que el $69 \%$ es casado y un $20 \%$ es soltero. De los 258 encuestados, 29 de ellos tenían la condición de separado o viudo.

\section{TABLA 3: Estado CIVIL Y díAs de tRABajo}

\begin{tabular}{lccccccccccc} 
Estado civil & $\begin{array}{c}\text { Frecuen- } \\
\text { cia }\end{array}$ & $\%$ & $\begin{array}{c}\text { Días de } \\
\text { trabajo }\end{array}$ & $\begin{array}{c}\text { Frecuen- } \\
\text { cia }\end{array}$ & $\%$ & Talca & Curico & Linares & $\begin{array}{c}\text { Cau- } \\
\text { quenes }\end{array}$ & $\begin{array}{c}\text { Parral } \\
\text { Consti- } \\
\text { tución }\end{array}$ \\
\hline Casado(a) & 179 & 69.4 & 1 & 4 & 1.6 & 53 & 23 & 27 & 24 & 24 & 28 \\
Soltero(a) & 49 & 19.0 & 2 & 36 & 14.0 & 16 & 9 & 7 & 7 & 5 & 5 \\
Viudo(a) & 12 & 4.7 & 3 & 25 & 9.7 & 2 & 4 & 2 & 2 & 1 & 1 \\
Separado (a) & 16 & 6.2 & 4 & 11 & 4.3 & 8 & 3 & 1 & 1 & 2 & 1 \\
Divorciada (a) & 1 & 0.4 & 5 & 12 & 4.7 & & & & & & 1 \\
\hline Total & 257 & 99.6 & 6 & 73 & 28.3 & 79 & 39 & 37 & 34 & 32 & 36 \\
Perdidos & 1 & 0.4 & 7 & 97 & 37.6 & & & & & & \\
\hline Total & 258 & 100.0 & Total & 258 & 100.0 & & & & & & \\
\hline
\end{tabular}

Fuente: Cuestionario final: antecedentes demográficos.

Trabajan mayoritariamente los siete días de la semana: un 28\% trabaja 6 días y un $14 \%$ sólo lo hace por 2 días. La media de días trabajados alcanza a 5.32. En general, un $65.9 \%$ de los comerciantes informales trabaja más de 5 días a la semana, y puesto que $\left(X^{2}{ }_{C}>X^{2}{ }_{t}\right)$ se rechaza la hipótesis nula, se puede afirmar que existe preferencia respecto de los días preferidos de trabajo. $\left(X^{2}{ }_{c}=209,567 ; X_{0.95,30 \text { g.l. }}=\right.$ $43,773)$ confirmándose de esta forma que el comercio informal se concentra principalmente los días sábado y domingo.

\section{Gráfico nº 2: ANTIGÜEDAD EN EL COMERCIO INFORMAL}

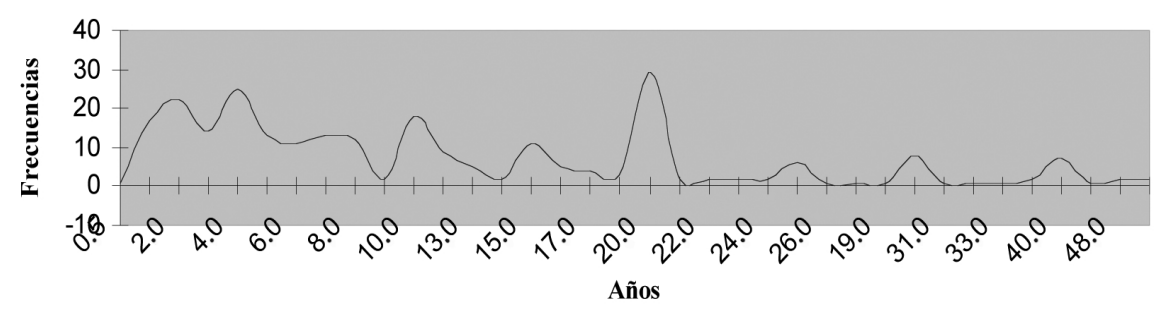

Fuente: Cuestionario final: antecedentes comerciales. 
La antigüedad en el comercio informal (Gráfico 2) alcanza una media de 12 años y la moda llega hasta 20 años de actividad. Un $85 \%$ de ellos se dedica de manera exclusiva al comercio ambulante, mientras el $14 \%$ de los encuestados relata tener otra fuente de ingresos. El 1\% restante no dio respuesta a esta pregunta. Por último, frente a la necesidad de búsqueda de un empleo, el $61 \%$ de los trabajadores manifiesta no sentir discriminación al momento de buscar trabajo (Tabla 4$)$.

TABLA 4: ReALIZACIÓN DE doS TRABAJOS Y GRADO DE DISCRIMINACIÓN

\begin{tabular}{lccccc} 
Dos trabajos & Frecuencia & $\%$ & Discriminación & Frecuencia & $\%$ \\
\hline Sí & 37 & 14.3 & Sí & 97 & 37.6 \\
No & 219 & 84.9 & No & 159 & 61.6 \\
\hline Total & 256 & 99.2 & Total & 256 & 99.2 \\
Perdidos & 2 & 0.8 & Perdidos & 2 & 0.8 \\
\hline Total & 258 & 100.0 & Total & 258 & 100.0 \\
\hline
\end{tabular}

Fuente: Cuestionario final: frecuencia de percepciones.

\section{Motivaciones, VENTAJAS Y LIMITACIONES DE LOS COMERCIANTES INFORMALES}

Lo que motiva a este tipo de "empresarios - trabajadores" a optar por esta actividad es preferentemente, el haber pasado por un largo periodo de cesantía, porque encuentran que el comercio informal es un negocio atractivo, privilegian obtener dinero en forma diaria, es un negocio familiar y es lo único que saben hacer (Tabla 5).

\section{TABLA 5: Motivos PARA ESTAR EN EL NEGoCiO Y DE NO PAGO DE IMPUESTOS}

\begin{tabular}{|c|c|c|c|c|c|}
\hline $\begin{array}{l}\text { Motivaciones para } \\
\text { estar en el negocio }\end{array}$ & Frecuencia & $\%$ & $\begin{array}{c}\text { Motivos de no Pago } \\
\text { de Impuestos }\end{array}$ & Frecuencia & $\%$ \\
\hline Largo periodo de cesantía & 75 & 29.1 & Falta de dinero & 25 & 9.7 \\
\hline Negocio atractivo & 45 & 17.4 & Burocracia para realizar trámites legales & 26 & 10.1 \\
\hline Trabajo familiar & 36 & 14.0 & Ahorro, al no pagar impuestos & 2 & 0.8 \\
\hline Lo único que sabe hacer & 20 & 7.8 & Desconocimiento de los trámites & 22 & 8.5 \\
\hline Flexibilidad de horarios & 10 & 3.9 & No le interesa & 34 & 13.2 \\
\hline Dinero en forma diaria & 42 & 16.3 & Está en trámite & 2 & 0.8 \\
\hline Incapacidad física & 15 & 5.8 & Total & 111 & 43.0 \\
\hline Otros & 15 & 5.8 & Perdidos & 147 & 57.0 \\
\hline Total & 258 & 100,0 & Total & 258 & 100.0 \\
\hline
\end{tabular}

Fuente: Cuestionario final: motivaciones comerciales. 
Tanto en hombres como en mujeres el principal motivo que los impulsa a este trabajo es el haber sufrido un largo periodo de cesantía. En el caso de las mujeres, un segundo motivo para optar por esta actividad es la posibilidad de generar dinero en forma diaria. Además, manifiestan no presentar conflictos con el comercio establecido y sólo un $21 \%$ de ellos reconoció que alguna vez ha debido dejar de trabajar por problemas con los propietarios del comercio establecido.

Respecto de las posibilidades de capacitación o de generar nuevos empleos, los encuestados manifiestan no tener acceso a capacitación y aunque un $96 \%$ afirmó que sólo quisieron generar su propio negocio, sin pensar en la generación de empleos, un $4 \%$ de los 258 encuestados tiene empleados a su servicio y de este porcentaje ninguno informó realizar contratos de trabajo.

Al referirse a su trabajo señalan en un $92 \%$ que es una ventaja, el $6 \%$ piensa que no lo es y un $2 \%$ de los consultados no respondió (Tabla 6). Respecto de la posesión de patente registrada en algún organismo público, un $80.2 \%$ dice estar registrado. El lugar donde mayoritariamente declaran tener algún tipo de registro para su negocio son las Municipalidades.

TABLA 6: Ventajas del COMERCIO INFORMAL Y REgistro de PERMisos

\begin{tabular}{|c|c|c|c|c|c|c|c|c|}
\hline Ventaja & Frecuencia & $\%$ & Registro & Frecuencia & $\%$ & Lugar de Registro & Frecuencia & $\%$ \\
\hline Sí & 237 & 91.9 & $\mathrm{Si}$ & 207 & 80.2 & Municipalidad & 180 & 69.8 \\
\hline NO & 16 & 6.2 & No & 51 & 19.8 & INP & 2 & 0.8 \\
\hline 3 & 1 & 0.4 & Total & 258 & 100.0 & Municipalidad y SII & 15 & 5.8 \\
\hline Total & 254 & 98.4 & & & & ACREMIC & 1 & 0.4 \\
\hline Perdidos & 4 & 1.6 & & & & S.F.I. & 1 & 0.4 \\
\hline \multirow[t]{4}{*}{ Total } & 258 & 100.0 & & & & S.I.I. & 8 & 3.1 \\
\hline & & & & & & Total & 207 & 80.2 \\
\hline & & & & & & Sin registro & 51 & 19.8 \\
\hline & & & & & & Total & 258 & 100.0 \\
\hline
\end{tabular}

Fuente: Cuestionario final: antecedentes de formalización de los comercios. 
Por otra parte, los encuestados que dicen no tener registro $(19.8 \%)$ señalan que se debe, principalmente, a que no les interesa registrarse. Manifiestan que esta situación de trabajo es producto del escaso interés por formalizar seguido de la falta de dinero, la burocracia que deben enfrentar y el desconocimiento de los trámites necesarios para lograrlo. Sólo un $1.8 \%$ dice estar en trámites para el pago de sus impuestos.

En general, los comerciantes informales no cuentan con ningún tipo de certificación ni inspecciones sanitarias (77\%), seguro laboral (96\%) y social (67\%). Del mismo modo el $99 \%$ dice no tener vacaciones pagadas (Tabla 7 ).

\section{TABLA 7: INSPECCIONES SANITARIAS Y SEGURIDAD LABORAL POR CIUDAD}

\begin{tabular}{lcccccc} 
& \multicolumn{3}{c}{ Inspecciones Sanitarias } & \multicolumn{3}{c}{ Seguridad Laboral } \\
\hline Ciudad & Sí & No & Total & Sí & No & Total \\
Talca & 10 & 70 & 80 & 1 & 78 & 79 \\
Curićó & 9 & 30 & 39 & 6 & 33 & 39 \\
Linares & 2 & 35 & 37 & 3 & 34 & 37 \\
Cauquenes & 10 & 24 & 34 & 2 & 32 & 34 \\
Parral & 11 & 21 & 32 & 3 & 29 & 32 \\
Constitución & 17 & 19 & 36 & 1 & 35 & 36 \\
\hline Total & 59 & 199 & 258 & 16 & 241 & 257 \\
\hline
\end{tabular}

Fuente: Cuestionario final: antecedentes comerciales.

En todas las ciudades se aprecia que mayoritariamente no cuentan con inspecciones sanitarias. Sólo en la ciudad de Constitución la variación es mínima entre tener o no tener registro de inspecciones sanitarias. Al consultar por seguro laboral se aprecia el mismo patrón de respuesta que en Talca donde un $70 \%$ no presenta ningún tipo de seguro laboral y el $98 \%$ no cuenta con previsión.

TABLA 8: SEguridAd SOCIAL, TIPO dE HORARIO Y UBICACIÓN FIJA POR CIUDAD

\begin{tabular}{lccccccccr} 
& \multicolumn{3}{c}{ Seguridad Social } & \multicolumn{3}{c}{ Tipo de horario } & \multicolumn{3}{c}{ Ubicación Fija } \\
Ciudad & Sí & No & Total & Sí & No & Total & Sí & No & Total \\
\hline Talca & 24 & 56 & 80 & 25 & 55 & 80 & 60 & 20 & 80 \\
Curicó & 15 & 24 & 39 & 14 & 25 & 39 & 34 & 5 & 39 \\
Linares & 8 & 29 & 37 & 18 & 19 & 37 & 31 & 6 & 37 \\
Cauquenes & 17 & 17 & 34 & 9 & 25 & 34 & 21 & 13 & 34
\end{tabular}


Economía Informal: un análisis al comercio ambulante de la región del Maule, Chile

\begin{tabular}{lccccccccc} 
Continiación TABLA & & & & & & & & & \\
Parral & 13 & 19 & 32 & 10 & 22 & 32 & 27 & 5 & 32 \\
Constitución & 9 & 27 & 36 & 11 & 25 & 36 & 31 & 5 & 36 \\
\hline Total & 86 & 172 & 258 & 87 & 171 & 258 & 204 & 54 & 258 \\
\hline
\end{tabular}

Fuente: Cuestionario final: antecedentes comerciales y de operación.

Además, la mayor parte de la población en estudio (66\%) señala no tener un horario fijo de trabajo, sin embargo, la mayoría $(79 \%)$ indicó tener un puesto y lugar fijo de trabajo. Del mismo modo, en todas las ciudades estudiadas la mayoría de los comerciantes ambulantes han tenido otro tipo de trabajo. En la ciudad de Constitución este predominio no es tan notorio (Tabla 8).

\section{TABLA 9: ReALIZACIÓN DE OtROS TRABAJOS Y CAMBIO dE TRABAJO POR CIUDAD}

\begin{tabular}{lcccccc} 
& \multicolumn{3}{c}{ Realiza Otros Trabajos } & \multicolumn{3}{c}{ Cambiaría de Trabajo } \\
Ciudad & Sí & No & Total & Sí & No & Total \\
\hline Talca & 68 & 12 & 80 & 42 & 37 & 79 \\
Curicó & 36 & 3 & 39 & 12 & 27 & 39 \\
Linares & 32 & 5 & 37 & 13 & 24 & 37 \\
Cauquenes & 20 & 13 & 33 & 11 & 23 & 34 \\
Parral & 22 & 10 & 32 & 8 & 24 & 32 \\
Constitución & 20 & 16 & 36 & 6 & 30 & 36 \\
\hline Total & 198 & 59 & 257 & 92 & 165 & 257 \\
\hline
\end{tabular}

Fuente: Cuestionario final: motivaciones comerciales.

Frente a la última pregunta (Tabla 9), acerca de si cambiaría su trabajo si recibe la misma remuneración, el 64\% de los encuestados dijo "no" estar dispuesto. La opinión de los trabajadores ambulantes de Talca difiere, mostrándose dispuestos al cambio. En Talca el $53 \%$ cambiaría su trabajo por uno establecido; por el contrario, en Constitución el 83\% no cambiaría su actual fuente laboral. Analizada la prueba de hipótesis respectiva y puesto que $\left(X^{2}{ }_{\mathrm{C}}>X^{2}{ }_{\mathrm{t}}\right.$, $)$ se rechaza la hipótesis nula, en consecuencia es posible afirmar que existe una relación entre las ciudades en estudio y la decisión de cambiarse a un trabajo formal remunerado $\left(X_{c}^{2}=18,335 ; X_{0.95,5}\right.$ g.l. $\left.=11,071\right)$. 


\section{NIVEL SOCIOECONÓMICO DE LOS COMERCIANTES INFORMALES}

Con relación al nivel educacional, un 54.7\% alcanzó estudios básicos, un $34.9 \%$ no los terminó y el $19.8 \%$ sí los finalizó. Menos de un $2 \%$ de los encuestados señaló no tener estudios. Un $41.5 \%$ declaró haber cursado enseñaza media pero sólo un $19.4 \%$ señaló haberlo terminado. Un $4 \%$ indicó tener estudios superiores y de ellos, cuatro $(1,5 \%)$ lograron un nivel profesional (Tabla 10).

\section{Tabla 10: Nivel educacional por CiUdad}

\begin{tabular}{lcccccccc} 
& Sin estudios & $\begin{array}{c}\text { Básica } \\
\text { incompleta }\end{array}$ & $\begin{array}{c}\text { Básica } \\
\text { completa }\end{array}$ & $\begin{array}{c}\text { Media } \\
\text { incompleta }\end{array}$ & $\begin{array}{c}\text { Media } \\
\text { completa }\end{array}$ & $\begin{array}{c}\text { Superior } \\
\text { incompleta }\end{array}$ & $\begin{array}{c}\text { Superior } \\
\text { completa }\end{array}$ & Total \\
\hline Frecuencia & 4 & 90 & 51 & 56 & 50 & 3 & 4 & 258 \\
\multicolumn{1}{c}{ Ciudad } & 1.6 & 34.9 & 19.8 & 21.7 & 19.4 & 1.2 & 1.6 & 100.0 \\
\hline Talca & 2 & 22 & 14 & 19 & 20 & 1 & 2 & 80 \\
Curico & 1 & 12 & 6 & 12 & 7 & & 1 & 39 \\
Linares & 11 & 13 & 8 & 4 & 1 & & 37 \\
Cauquenes & 1 & 14 & 6 & 3 & 9 & & 1 & 34 \\
Parral & 12 & 6 & 6 & 7 & 1 & & 32 \\
Constitución & & 19 & 6 & 8 & 3 & & & 36 \\
\hline Total & 4 & 90 & 51 & 56 & 50 & 3 & 4 & 258 \\
\hline
\end{tabular}

Fuente: Cuestionario final: antecedentes demográficos.

Respecto del ítem vivienda, un $68 \%$ afirma poseer casa propia y sólo un $12 \%$ manifiesta vivir de allegado (Tabla 11). Por otra parte, el ingreso diario por concepto de ventas es variable. Del total de encuestados, un $46 \%$ relata recibir menos de 5.000 pesos diarios equivalentes a US $\$ 8.33$ ( $\$ 600=\mathrm{US} \$ 1$ ), un $34 \%$ recibe entre 5.000 y 8.000 pesos y un $7 \%$ más de 15 mil pesos diarios, alcanzando los US\$ 25 (Tabla 12).

\begin{tabular}{lccccc} 
Ciudad & \multicolumn{5}{c}{ TABLA 11:TIPO DE VIVIENDA / CIUDAD } \\
& Arrendada & $\begin{array}{c}\text { Cedida por } \\
\text { familiares }\end{array}$ & Allegado & Casa propia & Total \\
\hline Talca & 10 & 2 & 15 & 53 & 80 \\
Curić & 5 & & 5 & 29 & 39 \\
Linares & 7 & & 4 & 26 & 37 \\
Cauquenes & 3 & 2 & 3 & 26 & 34 \\
Parral & 6 & 2 & 2 & 22 & 32 \\
Constitución & 9 & 4 & 2 & 21 & 36 \\
\hline Total & 40 & 10 & 31 & 177 & 258 \\
\hline
\end{tabular}

Fuente: Cuestionario final: antecedentes familiares. 
Cabe destacar que el ingreso diario varía según el género del comerciante. Así por ejemplo, en Talca reciben más dinero las mujeres, en cambio, en las otras ciudades de la Región, esta característica se revierte recibiendo mayores ingresos los hombres (Tabla 12).

TABLA 12: INGRESO DIARIO PROMEDIO TOTAL DE LA MUESTRA

\begin{tabular}{|c|c|c|c|c|c|}
\hline \multirow[b]{2}{*}{ Rango de Ingresos } & \multicolumn{2}{|c|}{ Ingresos Diarios } & \multicolumn{3}{|c|}{ Ingresos Diarios por Género } \\
\hline & Frecuencia & Porcentaje & Masculino & Femenino & Total \\
\hline $\begin{array}{l}\text { menos de } 5000 \\
\text { (menos de US\$ 8,3) }\end{array}$ & 119 & 46.1 & 54 & 65 & 119 \\
\hline $\begin{array}{l}\text { entre } 5000 \text { y } 8000 \\
\text { (US\$ } 8,3-13,3)\end{array}$ & 87 & 33.7 & 49 & 38 & 87 \\
\hline $\begin{array}{l}\text { entre } 8000 \text { y } 10.000 \\
\text { (US\$ } 13,3 \text { - 16,6) }\end{array}$ & 19 & 7.4 & 10 & 9 & 19 \\
\hline entre 10.000 y 12.000 (US\$ $16,6-20,0)$ & 13 & 5.0 & 6 & 7 & 13 \\
\hline entre 12.000 y 15.000 (US\$20,0-25) & 2 & .8 & 0 & 2 & 2 \\
\hline $\begin{array}{l}\text { más de } 15.000 \\
\text { (más US\$25,0) }\end{array}$ & 18 & 7.0 & 11 & 7 & 18 \\
\hline Total & 258 & 100.0 & 130 & 128 & 258 \\
\hline
\end{tabular}

Fuente: Cuestionario final: antecedentes comerciales.

El ingreso familiar mensual (Tabla 13) que reciben los encuestados se ubica en un rango bastante estrecho. Las mujeres relatan tener menos ingresos que los varones, exceptuando las que se desempeñan en la ciudad de Talca quienes superan a los hombres en sus ingresos. El promedio de ingresos mensual varía entre los US\$ 166.6 y US\$ 333.3 alcanzando, algunos de ellos (6.2\%) niveles superiores a los US\$500.

\section{TABLA 13: INGRESO FAMILIAR MENSUAL PROMEDIO POR GÉNERO}

\begin{tabular}{|c|c|c|c|c|c|c|}
\hline Género & $\begin{array}{l}\text { Menos de } \\
100.000 \\
\text { (US\$ 166,6) }\end{array}$ & $\begin{array}{l}\text { Entre } 100.000 \text { y } \\
150.000 \\
\text { (US\$ } 166,6-250)\end{array}$ & $\begin{array}{c}\text { Entre } 150.000 \\
\text { y } 200.000 \\
\text { (US\$250-333,3) }\end{array}$ & $\begin{array}{c}\text { Entre } 200.000 \\
\text { y } 300.000 \\
\text { (US\$333,3-500) }\end{array}$ & $\begin{array}{c}\text { Entre } 300.000 \\
\text { y } 400.000 \\
\text { (US\$ } \$ 500-666,6)\end{array}$ & $\begin{array}{l}\text { Más de } 400.000 \\
\text { (US\$ 666.6) }\end{array}$ \\
\hline Masculino & 25 & 57 & 25 & 14 & 4 & 5 \\
\hline Femenino & 52 & 32 & 23 & 14 & 5 & 2 \\
\hline Total & 77 & 89 & 48 & 28 & 9 & 7 \\
\hline
\end{tabular}

Fuente: Cuestionario final: antecedentes familiares. 


\section{Vi. Conclusiones}

En la Región del Maule, si bien su población ha crecido a una tasa del $6.2 \%$ promedio, no sucede lo mismo con el empleo que se ha visto impactado por el incremento de la fuerza de trabajo, por la incorporación de la mujer al empleo y por el incremento de la movilidad campo-ciudad. Los ocupados alcanzan a $90.9 \%$ y la tasa de desocupados (9.1\%) al año 2007. Un 8.8\% para los hombres y de un $9.7 \%$ para las mujeres. Sólo la ciudad de Linares supera los dos dígitos con un $11.7 \%$ de desempleados. Situación que, como se puede observar, confirma el modelo neoclásico de Solow (1956) según el cual, el crecimiento económico explica el comportamiento de indicadores claves como el empleo y cuyo corolario es que, a mayor inhabilidad del mercado para sostener dicho crecimiento mayor propensión al desarrollo de comercio informal. Situación que se verifica en el progresivo incremento de la cantidad de puestos de comercio informal que en la región alcanza un promedio por encima de 1.500 puntos de venta en un rango que va de los 900 a 2300 comercios. De ahí entonces que, efectivamente, la economía informal tiene su origen en sectores de baja productividad estancando el crecimiento (Brunner y Elecqua, 2003).

La Región del Maule presenta bajos niveles de escolaridad, confirmando las teorías del capital humano de Mincer, 1958, Schultz, 1961 y Becker 1964 (citados en Oroval y Escardíbul, op. Cit) para quienes la educación es una inversión que aumenta la dotación de capital e incrementa la riqueza, de manera que, en el caso de la Región del Maule, una baja escolaridad limita el crecimiento endógeno y estimula el comercio informal y contrae la riqueza (Cruz P., Rojas S., Vega G., 2002). Esta es una de las formas mediante las cuales se inicia un ciclo perverso que, sin duda, se explica por el bajo nivel de escolaridad. En este estudio, sólo un 54\% ha cursado el nivel básico de formación.

La síntesis no es precisamente promisoria. Los gestores del comercio ambulante de la Región del Maule, Chile, poseen un nivel educacional bajo, el 56.2\% de ellos no alcanzó estudios de enseñanza media, el 19.8\% termina la enseñanza básica, un 34.9\% la cursó en forma incompleta y un $1.6 \%$ no tiene estudios, afecta segmentos 
poblacionales cuya expresión por género es bastante homogénea con aproximadamente mitad de hombres y de mujeres que cumplen a su vez el rol de jefes de hogar.

Puesto que un $49 \%$ de los encuestados relató llevar más de 10 años dedicados a este tipo de negocio, destinando como mínimo 2 días de la semana a esta actividad y concurriendo en promedio 5 días, se confirma la existencia de una de las debilidades de la economía que, sobre la base de las teorías analizadas (Mincer, 1958, Schultz, 1961 y Becker 1964; citados en Oroval y Escardíbul, op. cit) existe una relación causal entre educación, productividad e ingresos. Así entonces, niveles de educación inferiores implican rentas bajas y contracción del crecimiento económico. Cabe destacar sin embargo, que un $36 \%$ de la población estudiada dice trabajar los siete días de la semana, lo cual sugiere un cierto grado de conformidad con los ingresos obtenidos en esta actividad. Sin perjuicio de lo cual, en general, no se sienten discriminados (61.6\%) al momento de buscar trabajo asalariado.

Respecto de la formalidad de sus actividades, un $80.2 \%$ indica estar inscrito en algún tipo de registro. Sin embargo, un 22\% esgrimió razones burocráticas para no formalizar plenamente sus actividades y, en un mismo porcentaje, expresó no contar con el dinero para estos efectos. Estas evidencias permiten confirmar la existencia actualizada de tianguis o comercios derivados de costumbres ancestrales (Lasso, 2000) que en el presente toman la forma de comercio callejero espontáneo sin regulación del Estado (Tijerina y Medellín, 1990) cuyos efectos son el no pago de impuestos y la precariedad de las relaciones laborales informales (OIT, 2003).

Sin duda, la formalización de actividades comerciales es considerada efectivamente una traba. Un $77.1 \%$ señaló no poseer inspecciones sanitarias, un $66.7 \%$ no dispone de seguro social y un altísimo $93.4 \%$ no posee seguro laboral. Quienes laboran en este sector están muy lejos de contar con un contrato de trabajo, participan de los denominados servicios informales (INEGI, 1997), son más bien independientes y trabajan fuera de la ley (Lasso, 2000) sin seguros social, laboral, vacaciones, ni aguinaldos, pero ahorran costos de acceso y reparto de utilidades (Hernández, 1997). Mas aún, sólo unos pocos $(4.3 \%)$ relatan tener empleados que trabajan a su servicio, 
sin embargo, reconocen tenerlos sin un contrato laboral generando informalidad, subempleo y accediendo a ingresos disminuidos (Jusidman, 1993), de lo cual se concluye y confirma la precariedad de estos empleos (Diamond y Mirrless, 1971).

En general, no reciben ningún tipo de capacitación y lo que les motiva a desempeñarse es, principalmente, la existencia de largos periodos de cesantía (29\%), disponibilidad diaria de dinero $(17 \%)$ y negocio atractivo (16\%) factores que dan sentido a la expresión de que "no son informales los hombres sino sus actividades" (Gershi, 1987), lo que se confirma a su vez, con un significativo $64 \%$ de entrevistados que indicó no querer cambiar de trabajo, aún cuando se les asegurara el mismo nivel de ingresos. Los ingresos diarios de los encuestados oscilan alrededor de los US\$ 8,3, lo que permite calcular un ingreso familiar promedio de entre US\$ 166.6 y US\$ 250 mensuales, en consecuencia, el nivel socioeconómico es bajo o medio bajo. Sin embargo, un $68 \%$ de los encuestados relata tener casa propia, lo que se puede explicar por el hecho de ser adultos con una edad promedio de 45 años y que, a esta edad, las familias ya han resuelto, aunque de manera modesta, su problema de vivienda.

Finalmente, respecto de "si cambiarían de trabajo" se aprecian importantes diferencias de opinión. En Talca la mayoría (53\%) responde que "sí". Por el contrario, en todas las demás ciudades se afirma que "no", confirmando que el comercio informal es un tipo de trabajo de alta aceptación, que proviene de la época pre colombina y que en la actualidad da forma a un estilo de vida entre quienes se dedican a esta actividad, preferentemente, en las ciudades más pequeñas de la Región del Maule de Chile. 


\section{Referencias}

Arzola, María Elenay, Rodrigo Castro. 2007. Determinantes de la Movilidad de la Pobreza en Chile (1196-2006). Programa Social de Libertad y Desarrollo, Serie Informe Social, N 112.

Brunner, J.J., Elacqua, G. 2003. Informe Capital Humano en Chile [en línea]. Universidad Adolfo Ibáñez, 2003 [fecha de consulta: 10 de mayo de 2008]. Disponible en: 〈http://www.aui.cl/p4_home/site/asocfile/ ASOCFILE120030528134> [fecha de consulta: 4 de junio de 2008].

Cruz P., Rojas S., Vega G., Villegas Y. 2002. El Capital Humano y la Gestión por Competencia [en línea]: documento electrónico en Internet. 2002 [fecha de consulta: 15 de junio de 2008]. Disponible en: <http:// www.mundotutorial.com/tutorial_el_capital_humano_por_competencias-mdtutorial343444.htm> [fecha de consulta: 4 de junio de 2008].

De Soto, Hernando. 1992. El Otro Sendero. ED. Sudamericana, Argentina.

Diamond, P., Mirrless, J. 1971.“Optimal Taxation and Public Production: I. Production Efficiency”. American Economic Review. Vol. 61, pp.: 8-27.

Doing Business 2009: Chile estancado en competitividad, Temas Públicos, $\mathrm{N}^{\circ}$ 886, 12 de septiembre de 2008; Libertad y Desarrollo, www.lyd. org [fecha de consulta: 4 de junio de 2008].

Gershi, Enrique. 1987. Economía informal en América latina. Cato Journal vol $7 \mathrm{n}^{-0} 1$.

Gershi, Enrique y De Soto, Hernando. 1987. El otro sendero: la revolución informal.

Hernández Licona, Gonzalo. 1997. The structure and recent evolution of the labour force in México. Documento de trabajo. ITAM.

Hernández, R., Fernández, C., Baptista, P. 1998. Metodología de la Investigación. Segunda Edición, Mc Graw- Hill. pp.60-62.

INE, Instituto Nacional de Estadística. Encuesta nacional de empleo, 2008.; www.INE.cl.

INE, Instituto Nacional de Estadística. Censo 2002. Mapa interactivo; www.censo2002.cl.

INEGI. 1997. Encuesta Nacional de Micro negocios.

Kinnear C., Tomas, Taylor R., James, 1993. Investigación de Mercado un enfoque aplicado. Cuarta Edición. Mc Graw-Hill, 812 p.; pp 128-129, pp 311 -. 319, pp 414-415, pp 450-451, pp. 812. 
Lasso, Pablo. 2000. El comercio informal; El comercio informal en países de América Latina. Reporte de investigación Academia Consumidor/Investigación de Mercados. ITESO; Año IV, Epoca1, Número 16, Marzo del 2000.

Martínez, R. 1999. Estrategias para sobrevivir. Inmigrantes en la venta ambulante. Intervención Psicosocial, 8 (2), 251-263.

MIDEPLAN, Casen. 2001 - 2006.

OIT. 2003. Documento de las Tendencias Mundiales del Empleo. pp:3.

OIT. 2003. Jacques Charmes, Women and Men in the Informal Economy: A Statistical Picture.

OIT. Ginebra. 2003. Tendencias mundiales del empleo.

OIT. 2002. 90 a Conferencia de la Organización Internacional del Trabajo.

OIT. 2002. Sector del empleo Documento de trabajo sobre la economía informal., Ginebra, p.4.

OIT. 2002. Como lo señala en su Informe VI "El trabajo decente y la economía informal" Conferencia Internacional del Trabajo de 2002.

OIT. 2002. Women and Men in the Informal Economy. A Statistical Picture, Ginebra, 88 pp 8-10.

OIT. Ginebra. 2002, El trabajo decente y la economía informal, Oficina Internacional del Trabajo.

Oroval, E. y Escardíbul, J. 2000. Aproximaciones a la relación entre educación y crecimiento económico. Revisión del Estado actual de la cuestión. [en línea] Universidad de Barcelona, 2000. Disponible en: <http://riscd2.eco. ub.es/ escard/Oroval-Escardibul.pdf $>$ [fecha de consulta: 4 de junio de 2008]

Pleitez, William, PNUD, 2008, Informe Nacional de Desarrollo Humano, El Salvador 2007-2008.

PREALC. 1988. Programa mundial del empleo. Mas allá de la regulación: El sector informal en América Latina, Primera edición. pp:14-16.

Programa Chile Califica. 2004. Análisis del comportamiento del mercado laboral en Chile, a través del procesamiento de información secundaria disponible.

Rodríguez, M. 1988. Economía subterránea. Tesis de grado, Pontificia Universidad Católica de Chile 181pp. 2-4.

Tanzi, V. 1983. "The underground economy". Finance and Development. Vol. 20, No. 4. IMF. Washington, D. C. Vol. 20 № 4.

Tijerina, J, Medellín, A. 1990. Análisis de difusión económica. Documento de Investigación No. 7. 
Economía informal: un análisis al comercio ambulante de la región del Maule, Chile

http://www.conama.cl/certificacion/1142/propertyvalue-12142.html [fecha de consulta: 6 de junio de 2008].

http://www.ilo.org/Search3/searchOnFast.do, [fecha de consulta: 9 de noviembre de 2008]

http://www.ilo.org/public/spanish/region/ampro/cintefor/temas/informal/tdecpob/index.htm [fecha de consulta: 10 de noviembre de 2008]. 
